\title{
Thermodynamic properties of a trapped Bose gas : A diffusion Monte Carlo study
}

\author{
S. Datta \\ S. N. Bose National Centre for Basic Sciences
}

September 11, 2018

\begin{abstract}
We investigate the thermodynamic properties of a trapped Bose gas of $\mathrm{Rb}$ atoms interacting through a repulsive potential at low but finite temperature $\left(k_{B} T<\mu<T_{c}\right)$ by Quantum Monte Carlo method based upon the generalization of Feynman-Kac method[1] applicable to many body systems at $\mathrm{T}=0$ to finite temperatures. In this letter we report temperature variation of condensation fraction, chemical potential, density profile, total energy of the system, release energy, frequency shifts and moment of inertia within the realistic potential model( Morse type ) for the first time by diffusion Monte Carlo technique. The most remarkable success was in achieving the same trend in the temperature variation of frequency shifts as was observed in JILA[2] for both $m=2$ and $m=0$ modes. For other things we agree with the work of Giorgini et al[3], Pitaevskii et al[4] and Krauth[5].
\end{abstract}




\section{Introduction}

After the experimental realization [6] of Bose Einstein Condensation and measurement[7] of thermodynamic quantities in alkali gases, there has been a growing interest in the theoretical study of these system since these could easily be modelled as systems with weakly interacting condensate and along with other things theoretical predictions about thermodynamical properties were possible in terms of simple quantum statistical mechanics. Most of these studies involve computational techniques to solve the relevant many body system and based on mean field theory such as the Gross Pitaevskii[8] technique. Despite their success in explaining the ground state properties, predictions in finite temperature properties become only approximate and often can lead to incorrect predictions as we have seen from the discripencies[9-11] between theory and experiment in explaining JILA top data. As a matter of fact mean field theory breaks down near $T_{c}$. It is therefore necessary to develop alternative computational methods which can solve these many body problems more accurately and rigorously.

Thermodynamics of Bose gases was studied before at a higher temperature $\left(k_{B} T>>\hbar \omega\right)$ by a semiclassical treatment[3]. Since effects of interactions become more pronounced at low temperatures we restrict our discussions at low but finite temperature $\left(k_{B} T<\mu<T_{c}\right)$. At low temperature the de Broglie wavelength of the atoms become appreciable, the study of thermodynamic behaviour at low temperatures ( of the order of harmonic oscillator temperature ) requires a quantum description of a lowlying elementary modes. As Quantum Monte Carlo technque and many body theory are closely connected, in this letter we present a quantum monte Carlo method namely Generalized Feynman-Kac method (GFK)[12,13] to study the thermodynamic properties of a Bose gas. From the equivalence of the imaginary time propagator and temperature dependent density matrix, finite tempera-

ture results can be obtained from the same zero temperature code by running it for finite time. The first Monte carlo calculations [5] on BEC deals with temperature dependence of condensation fraction and the other remarkable Monte Carlo calculation[14] deals with the ground state properties. We cal- 
culate temperature variation of condensation fraction, total energy, release energy, frequency shift, chemical potential and moment of inertia for system of $100 R b^{87}$ atoms.

\section{Theory}

All the thermodynamic quantities of interest are connected with the evaluation of eigensolution and eigenenergy of the the many body system. In this non mean field approach, we consider the full Hamiltonian for $100 R b^{87}$ atoms interacting through Morse potential at low but finite temperature and the solution of corresponding Schroedinger equation in path integral representation. We first describe the $\mathrm{T}=0$ version of the GFK formalism[12,13] and then generalize it to finite temperatures. For the Hamiltonian $H=-\Delta / 2+V(x)$ consider the initial value problem

$$
\begin{aligned}
i \frac{\partial u}{\partial t}= & \left(-\frac{\Delta}{2}+V\right) u(x, t) \\
& u(0, x)=f(x)
\end{aligned}
$$

with $x \in R^{d}$ and $u(0, x)=1$. The solution of the above equation can be written in Feynman-Kac representation as

$$
u(t, x)=E_{x} \exp \left\{-\int_{0}^{t} V(X(s)) d s\right\}
$$

where $\mathrm{X}(\mathrm{t})$ is a Brownian motion trajectory and $\mathrm{E}$ is the average value of the exponential term with respect to these trajectories. To speed up the convergence one can incorporate importance sampling in the underlying stochastic process and the lowest energy formula for eigenvalue for a given symmetry obtained from the large deviation priniciple of Donsker and Varadhan [15] can be written as

$$
\lambda=\lambda_{T}-\lim _{t \rightarrow \infty} \frac{1}{t} \ln E_{x} \exp \left\{-\int_{0}^{t} V_{p}(Y(s)) d s\right\}
$$

where $\mathrm{Y}(\mathrm{t})$ is the diffusion process which solves the stochastic differential equation

$$
d Y(t)=\frac{\Delta \psi_{T}(Y(t))}{\psi_{T}(Y(t))} d t+d X(t)
$$


The temperature dependence comes from the realization that the imaginary time propapagator is identical to the temperature dependent density matrix if $t \Rightarrow \beta=1 / T$ holds.

This becomes obvious when we consider the eqs[16]

$$
-\frac{\partial k(2,1)}{\partial t_{2}}=H_{2} k(2,1)
$$

and

$$
-\frac{\partial \rho}{\partial \beta}=H_{2} \rho(2,1)
$$

and compare

$$
k(2,1)=\sum_{i} \phi_{i}\left(x_{2}\right) \phi_{i}^{*}\left(x_{1}\right) e^{-\left(t_{2}-t_{1}\right) E_{i}}
$$

and

$$
\rho(2,1)=\sum_{i} \phi_{i}\left(x_{2}\right) \phi_{i}^{*}\left(x_{1}\right) e^{-\beta E_{i}}
$$

For Zero temp FK we had to extrapolate to $t \Rightarrow \infty$. For finite run time $\mathrm{t}$ in the simulation, we have finite temperature results. Here we show how to change our formalism to go from zero to finite temperature. We begin with the definition of finite temperature. A particular temperature ' $\mathrm{T}$ ' is said to be finite if $\Delta E<k T$ holds. The temperature dependent density matrix can be written in the following form

$$
\rho\left(x, x^{\prime}, \beta\right)=\rho^{(0)}\left(x, x^{\prime}, \beta\right) \times<\exp \left[-\int_{0}^{\beta} V_{p}[X(s)] d s\right]>_{D R W}
$$

At finite temperature thus free energy can be written as

$$
F=-\ln Z(x, \beta) / \beta=-\ln Z^{0}(x, \beta) / \beta-\ln <\exp \left[-\int_{0}^{\beta} V_{p}[X(s)] d s\right]>_{D R W} / \beta
$$




\subsection{The stationary state of the condensate}

To calculate the condensate energy and condensate density, we consider a cloud of $\mathrm{N}$ atoms interacting through repulsive potential and placed in a three dimensional anharmonic oscillator potential. We will assume that the condensate system has relaxed to a stationary state and at low energy the time independent Schroedinger equation representing the stationary state can be written as

$$
\left[-\Delta / 2+V_{\text {int }}+\frac{1}{2} \sum_{i=1}^{N}\left[x_{i}{ }^{2}+y_{i}{ }^{2}+\lambda z_{i}{ }^{2}\right]\right] \psi_{0}(\vec{r})=E \psi_{0}(\vec{r})
$$

where $\frac{1}{2} \sum_{i=1}^{N}\left[x_{i}{ }^{2}+y_{i}{ }^{2}+\lambda z_{i}^{2}\right]$ is the anisotropic potential with anisotropy factor $\lambda=\frac{\omega_{z}}{\omega_{x}}$. Now

$$
V_{i n t}=V_{\text {Morse }}=\sum_{i, j} V\left(r_{i j}\right)=\sum_{i<j} D\left[e^{-\alpha\left(r-r_{0}\right)}\left(e^{-\alpha\left(r-r_{0}\right)}-2\right)\right]
$$

Here we assume that the condensate oscillates in a static thermal bath. There is no interaction between the condensate and the thermal bath. The principal effect of finite temperature on the excitations is the depletion of condensate atoms. In the dilute limit and at very low energy only binary collisions are possible and no three body recombination is allowed. In such two body scattering at low energy first order Born approximation is applicable and the interaction strength ' $\mathrm{D}$ ' in the dimnsionless form $(\gamma)$ turns out to be

$$
\gamma=4 \frac{a \alpha^{3}}{s e^{\alpha r_{0}}\left(e^{\alpha r_{0}}-16\right)}=4.9 \times 10^{-5}
$$

For more details, one should look at [17]. In the above expression $a$ is the scattering length of $\mathrm{Rb}, \alpha$ is the width of the Morse potential, $r_{0}$ is the minimum of the potential well, 's' is the length scale in units of harmonic oscillator and $\lambda$ is the anisotropy factor. Here we have used[17] $\alpha=.29$ in harmonic oscillator units, $r_{0}=9.67$ in harmonic oscillator units, $a=$ $52 \times 10^{-10} \mathrm{~m}, s=.12 \times 10^{-5} \mathrm{~m}$ and $\lambda=\sqrt{8}$ For more details, one should look at [17]. 


\subsection{Effect of noncondensate}

In the case of noncondensate the system can be considered as a thermal gas. To calculate noncondensate energy and density we need to study the effect of noncondensate explicitly and consider the following stationary state for the thermal gas.

$$
\begin{gathered}
{\left[-\Delta / 2+2 V_{\text {int }}+V_{\text {trap }}\right] \psi_{j}(\vec{r})=E_{n c} \psi_{j}(\vec{r})} \\
{\left[-\Delta / 2+2 V_{\text {int }}+\frac{1}{2} \sum_{i=1}^{N}\left[x_{i}{ }^{2}+y_{i}^{2}+\lambda z_{i}^{2}\right] \psi_{j}\right](\vec{r})=E_{n c} \psi_{j}(\vec{r})}
\end{gathered}
$$

The basis wavefunction $\psi_{j}$ which describes the noncondensate should be chosen in such a way that it is orthogonal to $\psi_{0}$ as in Eq.(11) The most common way to achieve a orthogonal basis in Schroedinger prescription is to consider the dynamics of noncondensate in an effective potential[18,19] $V_{\text {eff }}=V_{\text {trap }}+2 V_{\text {int }}$. The factor 2 represents the exchange term between two atoms in two different states. The energy in the case of lowest lying modes then corresponds to $E=E_{c}+E_{n c}$. One can calculate the $E_{n c}$ using the same parameters as discussed in Sec 2.1 


\section{$3 \quad$ Results and discussions}

\subsection{The effect of temperature on frequency shifts}

\subsection{1 $\mathrm{m}=2$ mode}

Using Eq.(3), one can calculate the lowest lying energies due to any symmetry. To calculate the frequencies for $m=0$ and $m=2$ modes, one needs to find the energy differences of each of these states and the ground state. Underneath we show the data for frequency shifts for both $m=2$ and $m=0$ modes. For $m=2$ mode, considering motion of condensate only we achieve the downward shift of data all the way to $T=0.9 T_{c}$. But for the $m=0$ mode we need to consider the dynamics of thermal cloud also as discussed above.

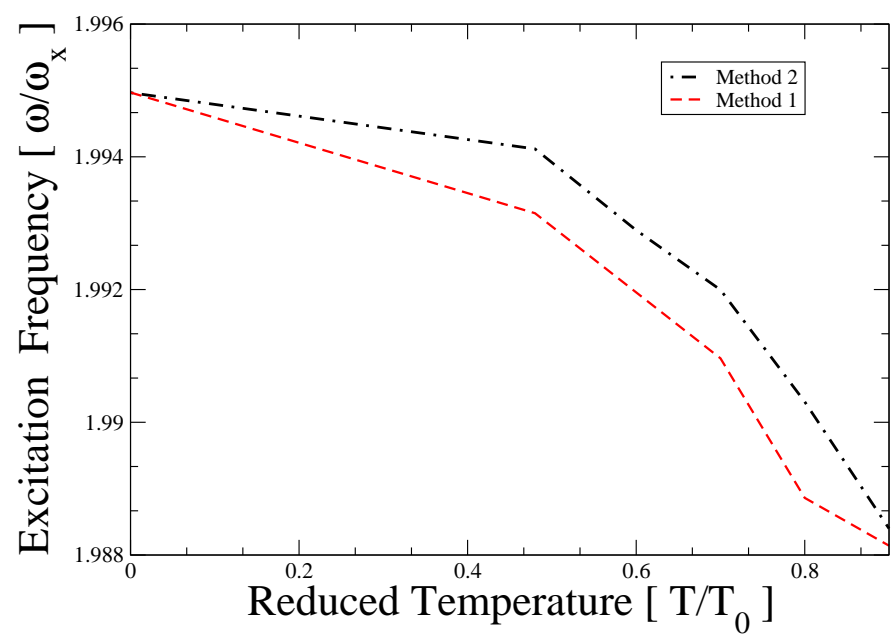

Figure 1: Effects of temperature on $\mathrm{m}=2$ mode; this work. The top cuve from equivalent $\mathrm{T}=0$ system[method 2], the bottom curve by putting temperature directly[method 1]. Both show agreement with JILA experimental data[2] all the way up to $0.9 T_{c}$ and $\operatorname{Ref}[20,21]$ 


\subsection{2 $\mathrm{m}=0$ mode}

For $m=0$ mode, considering the dynamics of condensate ( Sec 2.1) alone we do not get the upward shift as observed in JILA experimental data. But when the motion of thermal cloud (Sec 2.2) is considered in a dynamical manner, we observe the expected upward shift at around $T=0.7 T_{c}$.

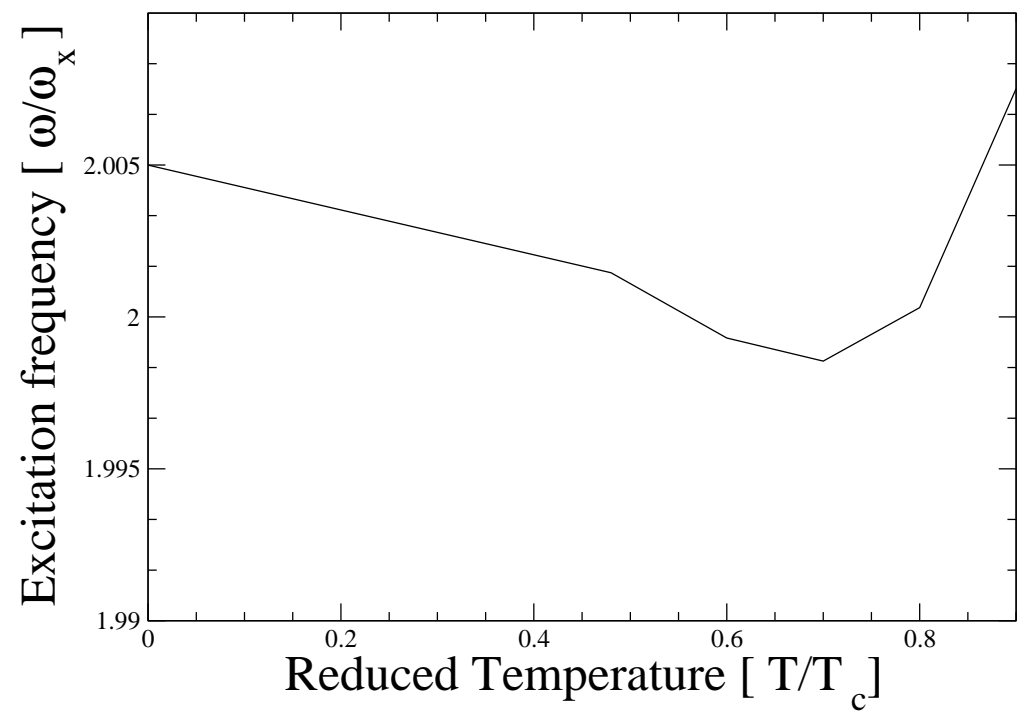

Figure 2: Effects of temperature on $\mathrm{m}=0$ mode from GFK considering noncondensate dynamics[this work], shows resemblence with JILA[2] and $\operatorname{Ref}[20,21]$

\subsection{The effect of temperature on condensate density}

The condensate density can be evaluated solving Eq.(11) and using Eq.(9). Underneath we plot the axial density due to condensate along $\mathrm{x}$ axis. 


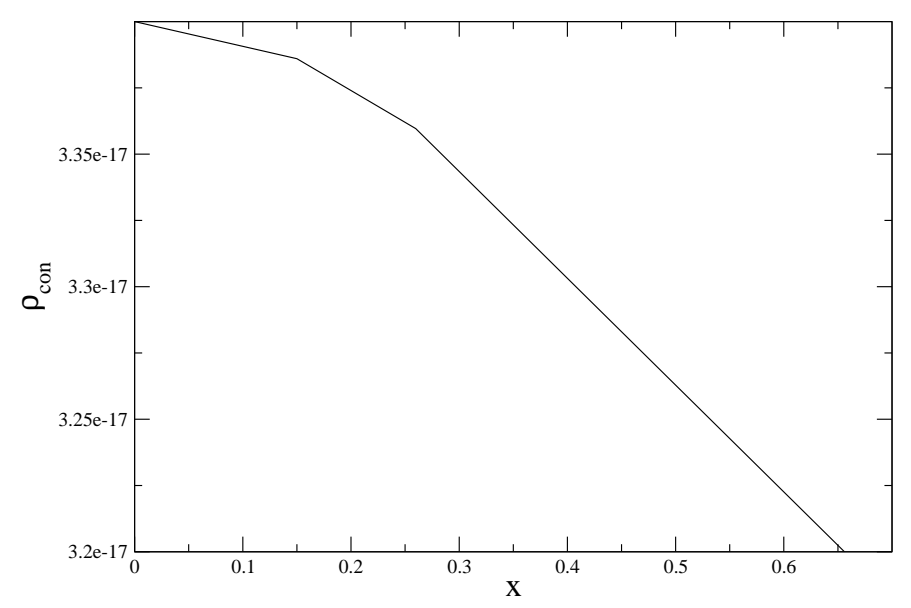

Figure 3: Axial density profile due to Condensate at temperature $\mathrm{T}=0.48$

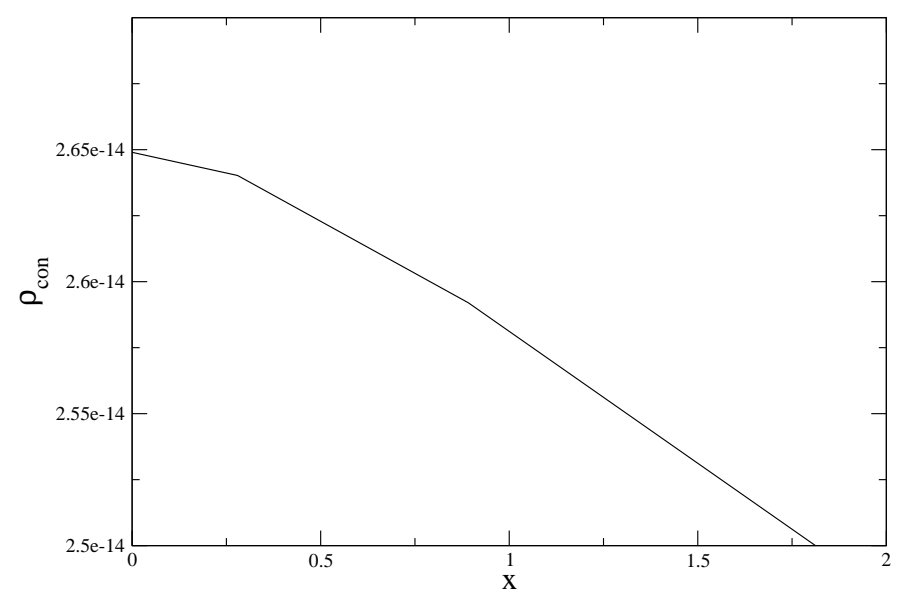

Figure 4: Axial density profile due to condensate at temperature $\mathrm{T}=0.6$

Fig 3, 4 and 5 are concerned with condensate density profile vs $\mathrm{x}$ for temperatures $T=0.48, T=0.6$ and $T=0.7$ respectively for a system of $100 \mathrm{Rb}$ atoms. We see that the center density for condensate increases as temperature is increased. This agrees with the earlier work of Krauth[5] 


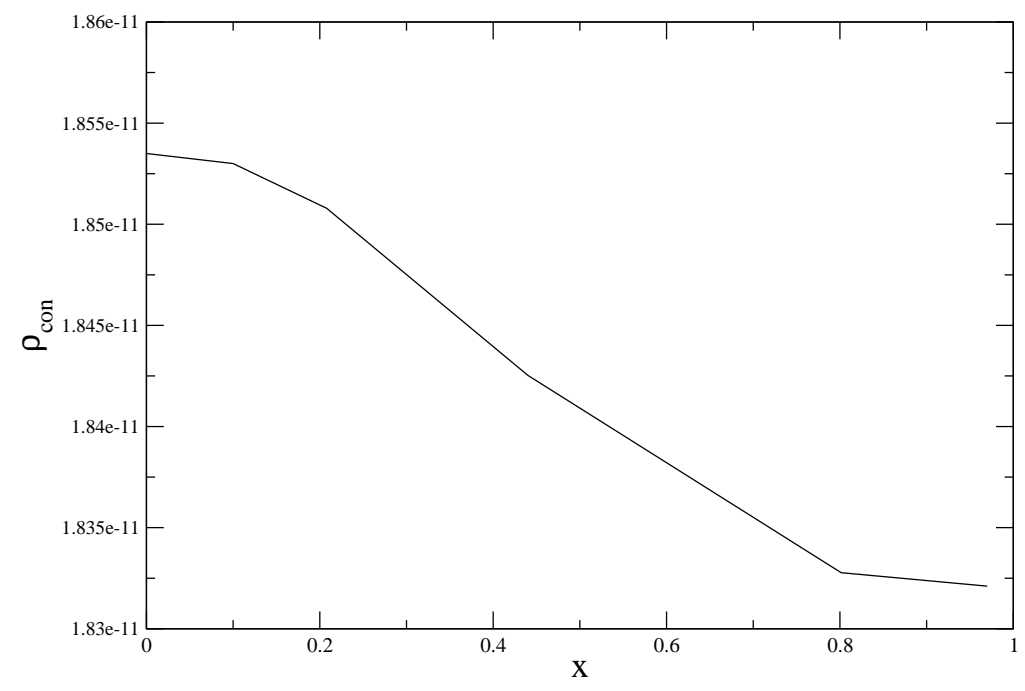

Figure 5: Axial density profile due to condensate at temperature $\mathrm{T}=0.7$

\subsection{The effect of temperature on the total density}

Following the theory in Sec 2.1 and 2.2 and using Eq.(9) one can calculate the total density of the Bose gas. Underneath we show the temperature variation of the total density along the $\mathrm{x}$ axis.

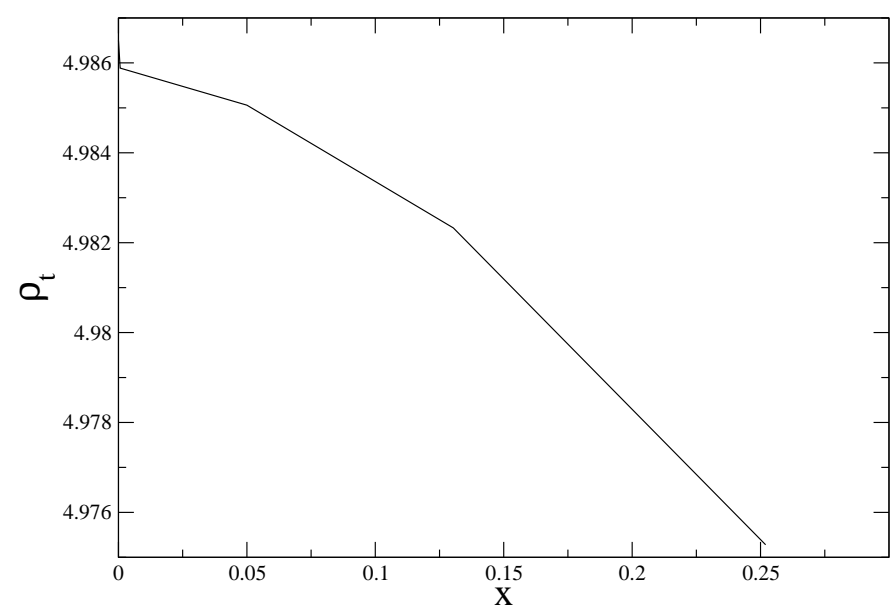

Figure 6: total density profile at temperature $\mathrm{T}=0.48$ 


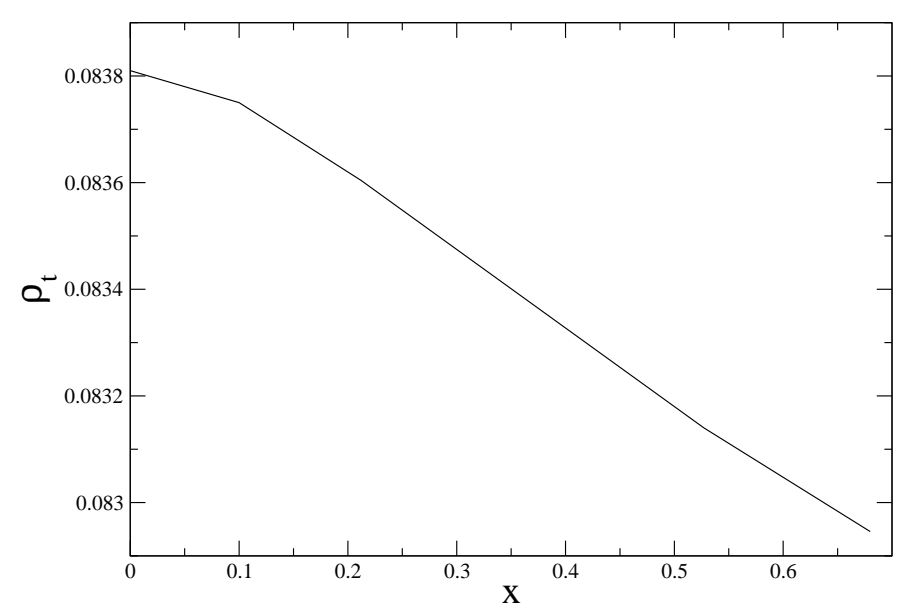

Figure 7: total density profile at temperature $\mathrm{T}=0.6$

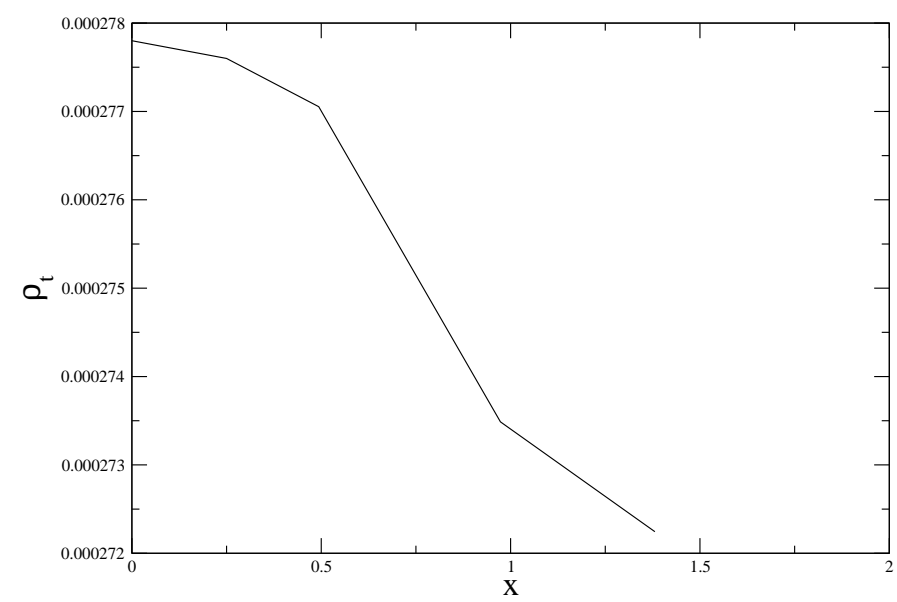

Figure 8: total density profile at temperature $\mathrm{T}=0.7$

On the other hand in Fig 6, 7 and 8 which represent the total density profile vs $\mathrm{x}$ at $T=.48, T=0.6$ and $T=0.7$ respectively, we see the opposite trend as the center density decreases as the temperature is raised. This also is in agreement with the ealier observation by Krauth[5] 


\subsection{Effect of temperature on the total energy of the Bose gas}

Total energy of the system The condensate and noncondensate energies can be evaluated solving Eq. (11) and Eq.(15) and using Eq(3). Then combining condensate and noncondensate energy we get the total energy of the system. The total energy of condensed and noncondensed component of a trapped Bose gas is a combination of $E_{k i n}, E_{h o}$ and $E_{\text {int }}$ for each component separately. The trend in the temperature variation of total energy is found to be the same as in $[3,4]$. In principle, specific heat can be calculated as the temperature derivative of total energy/particle keeping the confining potential constant. We will report it for bigger systems somewhere else. Release energy can be represented defined to be the energy obtained after switching off trap. We get similar trend the temperature variation of release energy as we see in $\operatorname{ref}[4]$

$E_{\text {rel }}=E_{\text {kin }}+E_{\text {int }}$

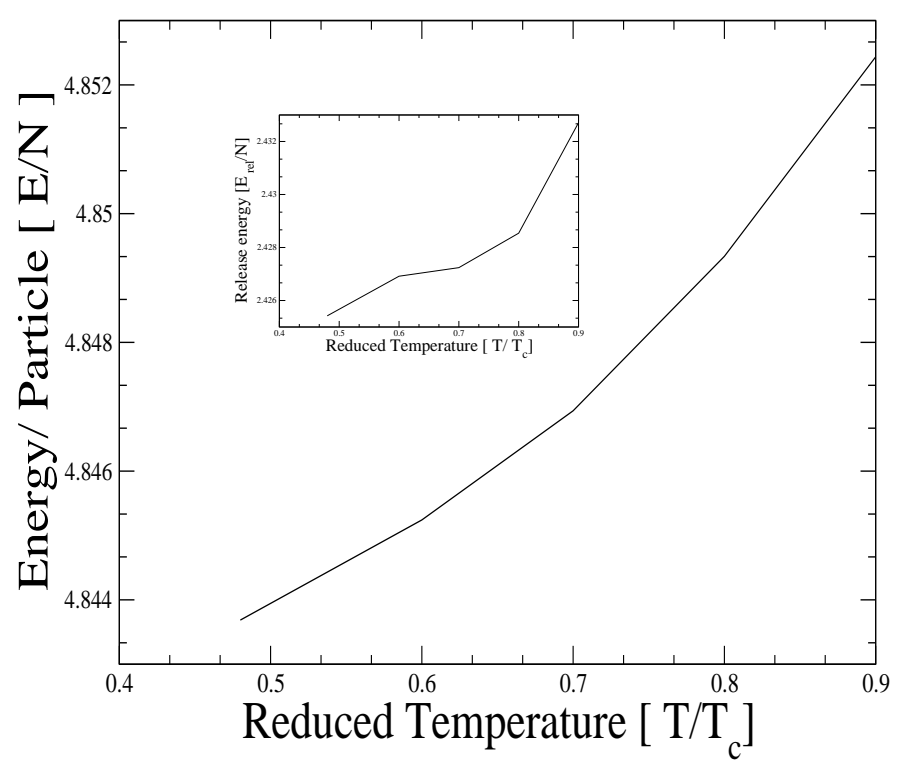

Figure 9: Total energy/particle as a function of reduced temperature. Inset: Release energy as a function of temperature 


\subsection{Effects of temperature on condensation fraction}

Interaction lowers the condensation fraction $\left(\frac{N}{N_{c}}\right)$ for repulsive potentials. Some particles always leave the trap because of the repulsive nature of the potential and on the top of it, if temperature is increased further, more particles will fall out of the trap and get thermally distributed.This decrease in condensation fraction eventually would cause the shifts in the critical temperature $\left(T_{c}\right.$ decreases $)$. We would observe this in (Fig.10). Earlier this was done by W. Krauth[5] for a large number of atoms by path integral Monte Carlo methed

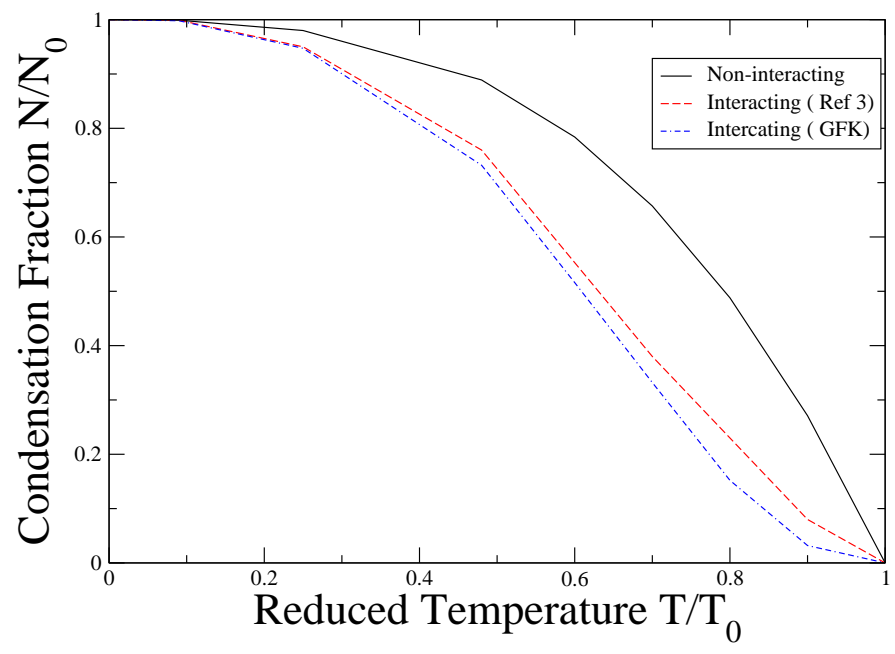

Figure 10: Condensation fraction vs Reduced Temperature ; this work. The innermost curve corresponds to the 100 interacting atoms and the outermost curve corresponds to the noninteracting case. The middle curve corresponds to the interactive case in ref[3]. The number of condensed particles decreases with the interaction 


\subsection{Effects of temperature on Chemical potential}

The chemical potential $\mu$ can be written in terms of different contributions to the energy, namely $E_{k i n}, E_{h o}$ and $E_{\text {int }}$ as follows[4].

$$
\mu=\frac{1}{N}\left(E_{k i n}+E_{h o}+2 E_{\text {int }}\right)
$$

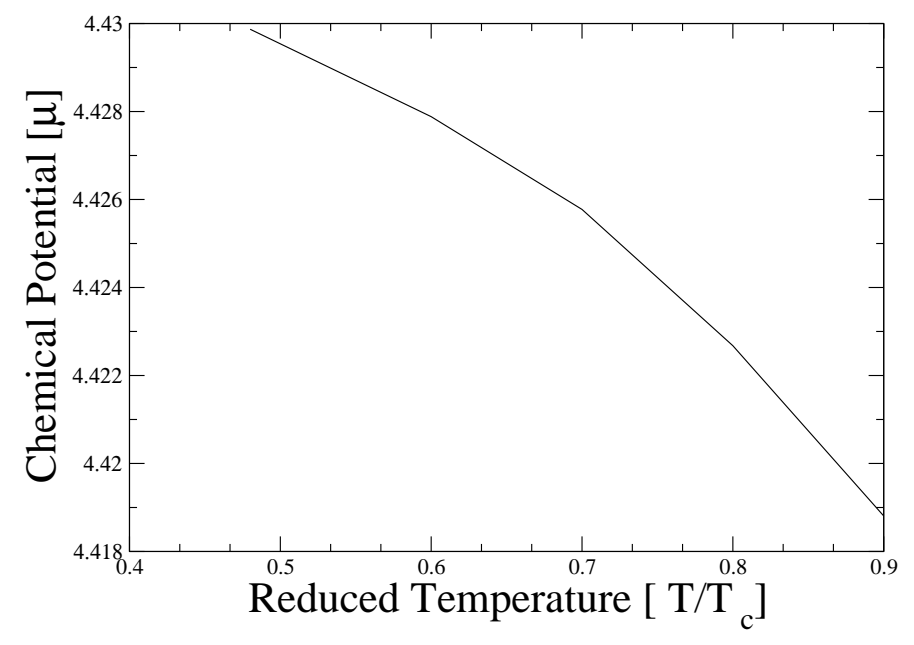

Figure 11: Chemical Potential vs Reduced Temperature 


\subsection{Effects of temperature on moment of inertia}

Moment of inertia The deviation of moment of inertia from its rigid value is given by the useful expression[22]

$$
\frac{\theta}{\theta_{\text {rig }}}=\frac{N_{T}<r^{2}>_{T}}{N_{0}<r^{2}>_{0}+N_{T}<r^{2}>_{T}}
$$

where $<>_{0}$ and $<>_{T}$ denote the average taken over the condensate and noncondensate densities of the Bose gas respectively.

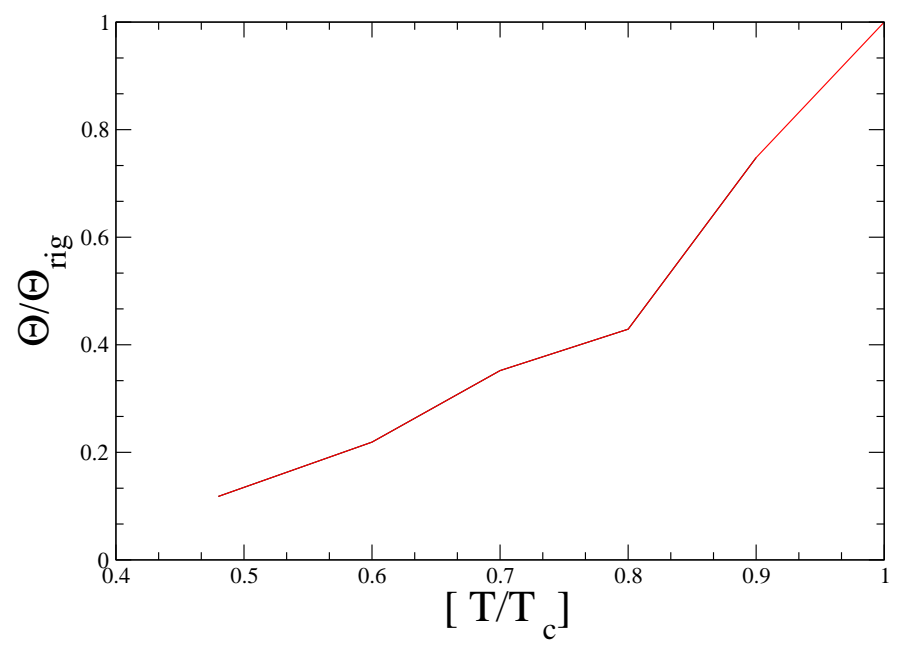

Figure 12: Moment of inertia $\theta$ divided by its rigid value $\theta_{\text {rig }}$ as a function of $T / T_{c}$

At $T=0, N_{T}=0$ and $\frac{\Theta}{\Theta_{r i g}}=0$. On the other hand fo $T=T_{c}$, $\frac{\Theta}{\Theta_{\text {rig }}}=1$ as $N_{0}=0$. 


\section{Conclusions:}

For the first time we have calculated finite temperature properties beyond meanfield approximation( GP etc) by Quantum Monte Carlo technique. We have calculated spectrum of $\mathrm{Rb}$ gas by considering realistic potentials like Morse potential etc. instead of conventional pseudopotentials for the first time. Since a dilute gas consisting of $R b^{87}$ atoms is a bosonic system the random walk in GFK is exact in the limit scale, time for walk and the number of walks get arbitraly large and it turns out that the this method is a potentially good candidate for a sampling procedure for Bose gases at all temperatures.

We are dealing with only 100 atoms. Nonetheless we have been able to show the variation of condensate and noncondensate density with temperature as a hallmark of BEC and lowering of condensation fraction in the case of interacting case which is very unique with a system of trapped atoms compared to uniform system.

In our non mean field study for finite temperature excitaion spectrum for $\mathrm{m}=2$ mode, we see agreement with experimental study all the way to $T=0.9 T_{c}$ [Fig. 1]. For $m=0$ mode, considering the motion of thermal cloud explicitly, we observe the upward shift of data[Fig 2] in JILA[2] and Ref[20,21]. Since at low temperature we solve the many body problem by considering the full Hamiltonian with realistic potential full quantum mechanically and nonperturbatively, obviously the above modes are collective in nature and correspond to the $\mathrm{m}=2$ and $\mathrm{m}=0$ experimental modes[7].

Since we are dealing with very small number of particles we cannot compare our data with other existing results directly on the same graph. We can neither use scaling method since we are working on a small system at a low temperature regime $k_{B}<\mu<T_{c}$. We can study and compare the temperature variation of different quantities such as total energy, release energy, moment of inertia with those in the literature[3,4,5] only qualitatively.

The method is extremely easy to implement and our fortran code at this point consists of about 270 lines. We employ an algorithm which is essentially parallel in nature so that eventually we can parallelize our code 
and calculate thermodynamic properties of bigger systems with the scaling property ( of the order of 2000 atoms ) taking advantage of the new computer architechtures This work is in progress. We are continuing on this problem and hope that this technique will inspire others to do similar calculations. 


\section{References}

[1] M. D. Donsker and M. Kac, J. Res. Natl. Bur. Stand, 44581 (1950), see also, M.Kac in Proceedings of the Second Berkley Symposium(Berkley Press, California, 1951 ), A. Korzeniowski, J. L. Fry, D.E. Orr and N. G. Fazleev, Phys. Rev. Lett. 69, 893,(1992)

[2] D. S. Jin, M. R. Mathews, J. R. Ensher, C. E. Wieman and E. A. Cornell Phys Rev Lett 78764 (1997)

[3] S. Giorgini, L. p. Pitaevskii and S. Stringari, Phys. Rev Lett. 783987 (1997), arXiv cond-mat/9704014 (1997).

[4] L. Pitaevskii and S. Stringari, Bose Einstein Condensation (Oxford University press) 2003

[5] W. Krauth, Phys. Rev Lett, 77 3695(1996)

[6] M. H. Anderson, J.R. Ensher, M.R. Matthews, C. E. Wieman E. A. Cornell, Science 269,198 (1995)

[7] M. O. Mewes, M. R. Andrews, N. J. van Druten, D. M. Kurn, D. S. Durfee and W. Ketterle. Phys. Rev. Lett 416(1996), J.R. Ensher, D. S. Jin, M.R.Mathews,, C. E. Wieman and E. A. Cornell Phys. Rev. Lett. 774984 (1996)

[8] V. L. Ginzburg and L.P. Pitaevski, Zh. Eksp. Teor Fiz, 34 1240(1958)[Sov. Phys. JETP 7, 858 (1958)], E.P. Gross, J. Math Phys.4, $195(1963)$

[9] R.J. Dodd, M.Edwards, C. W. Clark and K.Burnett 57, Phys Rev A,57 , R32 (1998).

[10] D. A. Hutchinson, R. J. Dodd ans K Burnett, Phys. Rev. Lett 81, 2198 $(1998)$

[11] H Shi and W. Zheng, Phys. Rev A 59, 1562 (1999) 
[12] M.Cafferel and P. Claverie, J. Chem Phys. 88 , 1088 (1988), 88, 1100 (1988)

[13] S. Datta, J. L Fry, N. G. Fazleev, S. A. Alexander and R. L. Coldwell, Phys Rev A 61 (2000) R030502, Ph. D dissertation, University of Texas at Arlington, 1996

[14] J. L DuBois, Ph D dissertation, University of Delaware,(2003).

[15] M. D. Donsker and S. R. Varadhan, in Proc. of the International Conference on Function space Integration ( Oxford Univ. Press 1975)pp. $15-33$

[16] Feynman And Hibbs, Quantum Mechanics and Path Integrals, ( McGraw-Hill, NY,1965 ).

[17] S. Datta,arXiv cond-mat/0603016(2006)

[18] R. J. Dodd, M. Edwards and C. W. Clark, J. Phys. B 32 4107-4115, 1999.

[19] S. A. Morgan, J.Phys. B 33,3847-3893, 2000

[20] S. A. Morgan, M.Rusch, D. A. W. Huchinson, K. Burnett, Phys. Rev Lett.,91, 250403, 2003

[21] B. Jackson and E. Zaremba, 88, 180402,2002

[22] F. Dalfovo, S. Giorgini, L.P. Pitaevskii and S. Stringari Rev Mod Phys 714631999 


\section{Acknowledgements:}

Financial help from DST ( under Young Scientist Scheme (award no. SR/FTP/-76/2001 )) is gratefully acknowledged. The author would like to thank Prof J. K. Bhattacharjee, Indian Association for the Cultivation of Science, India for suggesting the problem and many stimulating discussions. 